\title{
New records of mosquito species (Diptera: Culicidae) for Santa Catarina and Paraná (Brazil)
}

\author{
Gerson Azulim Müller ${ }^{1,3}$, Eduardo Fumio Kuwabara', Jonny Edward Duque ${ }^{1}$, \\ Mario Antônio Navarro-Silva ${ }^{1}$ \& Carlos Brisola Marcondes ${ }^{2}$ \\ ${ }^{1}$ Departamento de Zoologia, Universidade Federal do Paraná - UFPR, \\ CP 19020, CEP 81531-980, Curitiba, PR, Brazil, www.ufpr.br \\ ${ }^{2}$ Departamento de Microbiologia e Parasitologia, Centro de Ciências Biológicas, \\ Universidade Federal de Santa Catarina - UFSC, \\ CEP 88040-900, Florianópolis, SC, Brazil, www.ufsc.br \\ ${ }^{3}$ Corresponding author: Gerson Azulim Müller, e-mail: gecoazul@hotmail.com
}

MÜLLER, G.A., KUWABARA, E.F., DUQUE., J.E., NAVARRO-SILVA, M.A. \& MARCONDES, C.B. 2008. New records of mosquito species (Diptera: Culicidae) for Santa Catarina and Paraná (Brazil). Biota Neotrop. 8(4): http://www.biotaneotropica.org.br/v8n4/en/abstract?short-communication+bn01208042008.

Abstract: We provide eight new mosquito species records for Santa Catarina (Limatus flavisetosus Oliveira Castro 1935, Mansonia flaveola (Coquillett 1906), Ma. titillans (Walker 1848), Psorophora forceps Cerqueira 1939, Sabethes xyphydes Harbach 1994, Toxorhynchites bambusicolus (Lutz \& Neiva 1913), Tx. theobaldi (Dyar \& Knab 1906) and Wyeomyia lassalli Bonne-Wepster \& Bonne 1921) and three for Paraná (Ochlerotatus argyrothorax Bonne-Wepster \& Bonne 1920, Uranotaenia pallidoventer Theobald 1903 and Wyeomyia pilicauda Root 1928). Additionally, we list all species in these eight genera recorded previously in the two states. The known distribution and possible epidemiological implications of the new species records are discussed.

Keywords: Ochlerotatus, Psorophora, Sabethes, Toxorhynchites, Wyeomyia, Mansonia.

MÜLLER, G.A., KUWABARA, E.F., DUQUE, J.E., NAVARRO-SILVA, M.A. \& MARCONDES, C.B. 2008. Novos registros de espécies de mosquitos (Diptera: Culicidae) em Santa Catarina e Paraná (Brasil). Biota Neotrop. 8(4): http://www.biotaneotropica.org.br/v8n4/pt/abstract?short-communication+bn01208042008.

Resumo: Relatamos o primeiro encontro de oito espécies de mosquitos para Santa Catarina (Limatus flavisetosus Oliveira Castro 1935, Mansonia flaveola (Coquillett 1906), Ma. titillans (Walker 1848), Psorophora fórceps Cerqueira 1939, Sabethes xyphydes Harbach 1994, Toxorhynchites bambusicolus (Lutz \& Neiva 1913), Tx. theobaldi (Dyar \& Knab 1906) e Wyeomyia lassalli Bonne-Wepster \& Bonne 1921) e três para o Paraná (Ochlerotatus argyrothorax Bonne-Wepster \& Bonne 1920, Uranotaenia pallidoventer Theobald 1903 e Wyeomyia pilicauda Root 1928). Adicionalmente, apresentamos lista de todas as espécies destes oito gêneros com registro nos dois estados. A distribuição conhecida das espécies e sua possível importância epidemiológica são discutidas. Palavras-chave: Ochlerotatus, Psorophora, Sabethes, Toxorhynchites, Wyeomyia, Mansonia. 


\section{Introduction}

The dipteran family Culicidae includes several epidemiologically important insects, mostly related to the transmission of arboviruses and malaria parasites, but also to the annoyance caused by their bites (Consoli \& Oliveira 1994). The Brazilian southern states of Santa Catarina and Paraná are included in the geographical distribution of several arboviruses (Forattini 2002), but information on the mosquito fauna is mostly restricted to those species related to malaria transmission, such as Anopheles (Kerteszia) spp. in Santa Catarina (e.g. Rachou \& Ferraz 1951, Ferreira 1965) and Anopheles (Nyssorhynchus) spp. in Paraná (e.g. Guimarães et al. 1997). Knowledge of the distribution of mosquito species is essential for understanding the risks to human and animal health and the development of future control measures. The objective of the present paper is to present new records of several mosquito species of eight genera and list species in these genera previously recorded for Paraná and Santa Catarina states.

\section{Methods}

Mosquitoes were collected in nine different localities of Santa Catarina and Paraná States between 1999 and 2008 (Table 1). All localities belong to the Atlantic Forest biome, and are constituted by dense ombrophilous forest. The climate is tropical, with high temperatures (means higher than $22^{\circ} \mathrm{C}$ ) and high rain precipitation, well distributed thorough the year (Veloso et al. 1991). Except for Unidade de Conservação Ambiental Desterro (Florianópolis, SC), Floresta Nacional (Ibirama, SC) and Floresta do Palmito (Paranaguá, PR), all the studied localities are constituted by forest fragments smaller than 10 ha, highly influenced by human activities, either by agriculture or by the proximity of urban areas. All the forest fragments had several breeding places for mosquitoes, mostly phytotelmata (e.g. bromeliads), shallow pools on rocks and, in Ascurra, Indaial and Ibirama, marshes with aquatic plants.

Five different methodologies were utilized for the daylight collections: Shannon trap without light, CDC-like trap baited by a hen, artificially drilled bamboo internodes, attracted by humans and collected by suction tubes and aspiration from the vegetation. Mosquitoes were identified using descriptions and keys of Lane (1953a, b), Correa \& Ramalho (1956), Consoli \& Oliveira (1994) and Forattini (2002). When necessary, specimens were compared with those of the Faculdade de Saúde Pública da Universidade de São Paulo (São Paulo, Brazil). All specimens were glued to card triangles on entomological pins and are located in the Departamento de Microbiologia e Parasitologia da Universidade Federal de Santa Catarina and in the Entomological Collection Padre Jesus Santiago Moure, Departamento de Zoologia, Universidade Federal do Paraná (DZUP). Genera and subgenera of Culicidae are abbreviated according to Reinert (1975), and Ochlerotatus was considered a valid genus (Reinert 2000), as recommended by Marcondes (2007). The subgenus Ochlerotatus is abbreviated as Och. according to Reinert et al. (2004).

\section{Results and Discussion}

Eleven species were recorded for the first time for the states of Santa Catarina and Paraná (Table 2). These new records are distributed in eight genera. A review of the literature indicated that 89 species included in these eight genera have been previously recorded in Paraná and Santa Catarina states (Table 3).

Mansonia includes two subgenera: Mansonia, not recorded in the Neotropical Region, and Mansonioides, which had 15 species recorded from this region (Barbosa et al. 2007). From these, Ma. titillans (Walker, 1848) and Ma. flaveola (Coquillett, 1906) are widely distributed, from southern United States (Florida and Texas) to extreme south of Brazil, Argentina and Uruguay (Heinemann \& Belkin 1977, Cardoso et al. 2005). Mansonia titillans (Walker 1848) was previously reported as vector of Venezuelan Encephalitiditis Virus (Méndez et al. 2001).

Ochlerotatus includes 58 species in Central and South America (Reinert et al. 2005). The larvae of Oc. argyrothorax BonneWepster \& Bonne 1920 develop in tree holes and shallow ponds on rocks (Pereira et al. 2005). Psorophora forceps Cerqueira, 1939 had been recorded in Mato Grosso do Sul, Espírito Santo and Rio de Janeiro states (Guedes et al. 1965). The present report from Santa Catarina considerably extends its known distribution. Uranotaenia pallidoventer Theobald, 1903 was reported in Amazonian Forest (Hutchings et al. 2005). It should be emphasized the absence of previous reports of Uranotaenia from Santa Catarina (Table 3).

Among species developing in phytotelmata, Sa. xhyphides Harbach, 1994 had been reported for two municipalities of Rio Grande do Sul, the most southern state of Brazil (Cardoso et al. 2005). Since the mosquitoes referred by Prado (1935) in bamboos at São Paulo state as Sa. intermedius (Lutz, 1904) were considered as Sa. xhyphydes (Harbach 1994), Santa Catarina would be the third state to have this species reported.

The distribution of Toxorhynchites (Lynchiella) includes, besides tropical regions, also other subtropical and temperate ones, differently from the other two subgenera (Collins \& Blackwell 2000). Toxorhynchites theobaldi (Dyar \& Knab 1906), besides occurring in bamboo internodes, can also be found in tree holes (Silva \& Lozovei 1999).

The present report adds to the recent reports by Paterno \& Marcondes (2004), Marcondes et al. (2003, 2006) and Müller \&

Table 1. Localities of Santa Catarina and Paraná states where mosquitoes were collected.

Tabela 1. Localidades dos Estados de Santa Catarina e Paraná onde os mosquitos foram coletados.

\begin{tabular}{|c|c|c|}
\hline Locality & Coordinate & Altitude (a.s.l.) \\
\hline Ascurra, SC & $26^{\circ} 57^{\prime} 07^{\prime \prime} \mathrm{S}$ and $49^{\circ} 22^{\prime} 14^{\prime \prime} \mathrm{W}$ & $86 \mathrm{~m}$ \\
\hline Floresta Nacional, Ibirama, SC & $27^{\circ} 02^{\prime} 23^{\prime \prime} \mathrm{S}$ and $49^{\circ} 27^{\prime} 29^{\prime \prime} \mathrm{W}$ & $330 \mathrm{~m}$ \\
\hline Floresta do Palmito, Paranaguá, PR & $25^{\circ} 31^{\prime} 13^{\prime \prime} \mathrm{S}$ and $48^{\circ} 30^{\prime} 34^{\prime \prime} \mathrm{W}$ & $2 \mathrm{~m}$ \\
\hline Governador Celso Ramos, SC & $27^{\circ} 18^{\prime} 53^{\prime \prime} \mathrm{S}$ and $48^{\circ} 33^{\prime} 33^{\prime \prime} \mathrm{W}$ & $40 \mathrm{~m}$ \\
\hline Guabiruba, SC & $27^{\circ} 05^{\prime} 09^{\prime \prime} \mathrm{S}$ and $48^{\circ} 58^{\prime} 52^{\prime \prime} \mathrm{W}$ & $60 \mathrm{~m}$ \\
\hline Indaial, SC & $26^{\circ} 53^{\prime} 39^{\prime \prime} \mathrm{S}$ and $49^{\circ} 13^{\prime} 34^{\prime \prime} \mathrm{W}$ & $82 \mathrm{~m}$ \\
\hline Nova Trento, SC & $27^{\circ} 15^{\prime} 49^{\prime \prime} \mathrm{S}$ and $48^{\circ} 55^{\prime} 22^{\prime \prime} \mathrm{W}$ & $379 \mathrm{~m}$ \\
\hline Unidade de Conservação Ambiental Desterro, Florianópolis, SC & $27^{\circ} 31^{\prime} 51^{\prime \prime} \mathrm{S}$ and $48^{\circ} 30^{\prime} 44^{\prime \prime} \mathrm{W}$ & $50-150 \mathrm{~m}$ \\
\hline
\end{tabular}


Mosquitos of Santa Catarina and Paraná

Table 2. New records of Culicidae species from the Brazilian southern states of Santa Catarina and Paraná.

Tabela 2. Novos registros de espécies de Culicidae para os Estados do sul brasileiro de Santa Catarina e Paraná.

\begin{tabular}{|c|c|c|c|}
\hline Species & Locality & Quantity/sex & Date \\
\hline \multicolumn{4}{|l|}{ Aedini } \\
\hline Ochlerotatus argyrothorax & Paranaguá, PR, (FEP) & 1 우 & 29.VI.2005 \\
\hline \multicolumn{4}{|l|}{ Bonne-Wepster \& Bonne, 1920} \\
\hline Psorophora forceps Cerqueira, 1939 & Guabiruba, SC & 1 우 & 13.X.1998 \\
\hline \multicolumn{4}{|l|}{ Mansoniini } \\
\hline Mansonia flaveola (Coquillett, 1906) & Ascurra, SC & 1 우 & 14.XI.1999 \\
\hline \multirow[t]{2}{*}{ Ma. titillans (Walker, 1848) } & Indaial, SC & $3 q$ & 10.V.1999 \\
\hline & Rio do Sul, SC & 2 우 & 20.X.1999 \\
\hline \multicolumn{4}{|l|}{ Sabethini } \\
\hline Limatus flavisetosus Oliveira Castro, 1935 & Ibirama, SC, (FLONA) & $1+9$ & 02.IV.2008 \\
\hline Sabethes xyphydes Harbach, 1994 & Florianópolis,SC, (UCAD) & $1 \hat{\delta}$, from immature forms & 22.V.2005 \\
\hline Wyeomyia lassalli & Florianópolis,SC, (UCAD) & $1+$ & 12.IV.2007 \\
\hline \multicolumn{4}{|l|}{ Bonne-Wepster \& Bonne, 1921} \\
\hline \multirow{10}{*}{ Wy. pilicauda (Root, 1928) } & Paranaguá, PR, (FEP) & 17 우 & 26.I.2005 \\
\hline & & 90 우 & 16.II.2005 \\
\hline & & 13 우 & 30.III.2005 \\
\hline & & $2 q$ & 27.IV.2005 \\
\hline & & 2 우 & 23.V.2005 \\
\hline & & 20 & 24.VIII.2005 \\
\hline & & 2 우 & 23.IX.2005 \\
\hline & & 5 우 & 31.X.2005 \\
\hline & & $2+$ & 23.XI.2005 \\
\hline & & 12 우 & 29.XII.2005 \\
\hline \multicolumn{4}{|l|}{ Uranotaeniini } \\
\hline \multirow{2}{*}{ Uranotaenia pallidoventer Theobald, 1903} & Paranaguá, PR, (FEP) & 3 우 & 30.X.2005 \\
\hline & & 1 우 & 23.XI.2005 \\
\hline \multicolumn{4}{|l|}{ Toxorhynchitini } \\
\hline $\begin{array}{l}\text { Toxorhynchites bambusicolus } \\
\text { (Lutz \& Neiva, 1913) }\end{array}$ & Florianópolis, SC, (UCAD) & $2 \hat{\sigma}$, from immature forms & 25.II.2006 \\
\hline Tx. theobaldi (Dyar \& Knab, 1906) & Florianópolis, SC, (UCAD) & $1 \hat{\jmath}$, from immature forms & 14.II.2006 \\
\hline
\end{tabular}

Table 3. Species of Ochlerotatus, Psorophora, Mansonia, Limatus, Sabethes, Wyeomyia, Uranotaenia and Toxorhynchites reported in Santa Catarina and/or Paraná states.

Tabela 3. Espécies de Ochlerotatus, Psorophora, Mansonia, Limatus, Sabethes, Wyeomyia, Uranotaenia e Toxorhynchites registrados para os Estados de Santa Catarina e/ou Paraná.

\begin{tabular}{|c|c|c|c|}
\hline Species & Santa Catarina & Paraná & References \\
\hline \multicolumn{4}{|l|}{ Aedini } \\
\hline $\begin{array}{l}\text { Ochlerotatus (Ochlerotatus) albifasciatus } \\
\text { (Macquart 1838) }\end{array}$ & $\mathrm{X}$ & & marcondes et al. (2003) \\
\hline Oc. (Och.)crinifer (Theobald 1903) & & $\mathrm{X}$ & $\begin{array}{l}\text { Barbosa et al. (2003), Consolim et al. (1993), Lopes \& Lozovei } \\
\text { (1995), Lopes (2002a), Tissot \& Silva (2008) }\end{array}$ \\
\hline Oc. (Och.) fluviatilis (Lutz 1904) & $\mathrm{X}$ & $\mathrm{X}$ & $\begin{array}{l}\text { Barbosa et al. (1993), Bona \& Navarro-Silva (2008), Consolim } \\
\text { et al. (1993), Guimarães et al. (2003), Lopes et al. (1993, } \\
\text { 1995b), Lopes \& Lozovei (1996), Lopes (1997b, c, 2002a, b), } \\
\text { Marcondes et al. (2006), Silva (2002), Tissot \& Silva (2008) }\end{array}$ \\
\hline Oc. (Och.) fulvus (Wiedemann 1828) & $\mathrm{X}$ & $\mathrm{X}$ & $\begin{array}{l}\text { Consolim et al. (1993), Guimarães et al. (2003), Marcondes } \\
\text { et al. (2003), Tissot \& Silva (2008) }\end{array}$ \\
\hline Oc. (Och.) hastatus Dyar 1922 & & $\mathrm{x}$ & Tissot \& Silva (2008) \\
\hline Oc. (Och.) hortator (Dyar \& Knab 1907) & & $\mathrm{X}$ & Bona \& Navarro-Silva (2008) \\
\hline Oc. (Och.) nubilus Theobald 1903 & & $\mathrm{X}$ & Tissot \& Silva (2008) \\
\hline Oc. (Och.) oligopistus Dyar 1918 & & $\mathrm{X}$ & Tissot \& Silva (2008) \\
\hline Oc. (Och.) pennai (Antunes \& Lane 1938) & & $\mathrm{X}$ & Guimarães et al. (2003) \\
\hline Oc. (Och.) rhyacophilus Costa Lima 1933 & $\mathrm{X}$ & $\mathrm{X}$ & $\begin{array}{l}\text { Marcondes et al. (2003), Santos-Neto \& Lozovei (2008), } \\
\text { Tissot \& Silva (2008) }\end{array}$ \\
\hline Oc. (Och.) scapularis (Rondani 1848) & $\mathrm{x}$ & $\mathrm{X}$ & $\begin{array}{l}\text { Barbosa et al. (2003), Bona \& Navarro-Silva (2008), Guimarães } \\
\text { et al. (2003), Lopes et al. (1995b), Lopes \& Lozovei (1996), } \\
\text { Marcondes \& Paterno (2005), Paterno \& Marcondes (2004), } \\
\text { Sant'Ana \& Lozovei (2001), Silva \& Menezes (1996), Silva } \\
\text { (2002), Tissot \& Navarro-Silva (2004) }\end{array}$ \\
\hline
\end{tabular}


Table 3. Continued...

\begin{tabular}{l}
\hline Species \\
\hline Oc. (Och.) serratus (Theobald 1901) \\
Oc. (Och.) stigmaticus (Edwards 1922) \\
Oc. (Och.) terrens (Walker 1856)
\end{tabular}

Psorophora (Grabhamia) cingulata (Fabricius 1805)

Ps.( Gra.) confinnis (Fabricius 1805)

Ps. (Janthinosoma) albipes (Theobald 1907)

Ps. (Jan.) albigenu (Lutz 1908)

Ps. (Jan.) circumflava Cerqueira 1943

Ps. (Jan.) discrucians (Walker 1956)

Ps. (Jan.) ferox (Humboldt 1819)

Ps. (Jan.) johnstonii (Grabham 1905)

Ps. (Jan.) lanei Shannon \& Cerqueira 1943

Ps. (Jan.) lutzii (Theobald 1901)

Ps. (Jan.) pseudomelanota

Barata \& Cotrim 1971

Ps. (Jan.) varipes (Coquillett 1904)

Ps. (Psorophora) ciliata (Fabricius 1794)

Ps. (Ps.) saeva Dyar \& Knab 1906

Mansoniini

Mansonia (Mansonia) amazonensis

(Theobald 1901)

Ma. (Man.) cerqueirai

(Barreto \& Coutinho 1932)

Ma. (Man.) flaveola (Coquillett 1906)

Ma. (Man.) fonsecai (Pinto 1932)

Ma. (Man.) humeralis Dyar \& Knab 1916

Ma. (Man.) iguassuensis

Barbosa, Navarro-Silva \& Sallum 2007

Ma. (Man.) indubitans Dyar \& Shannon 1925

Ma. (Man.) pessoai

(Barreto \& Coutinho 1944)

Ma. (Man.) pseudotitillans (Theobald 1901)

Ma (Man.) titillans (Walker 1848)

Ma. (Man.) wilsoni

(Barreto \& Coutinho 1944)

Sabethini

Santa Catarina Paraná References

$\mathrm{X}$

Barbosa et al. (1993, 2003), Bona \& Navarro-Silva (2008), Consolim et al. (1993), Guimarães et al. (2003), Marcondes et al. (2006), Paterno \& Marcondes (2004), Santos-Neto \& Lozovei (2008), Tissot \& Navarro-Silva (2004)

x Barbosa et al. (1993)

$\mathrm{X}$

$\mathrm{x}$

Barbosa et al. (1993), Consolim et al. (1993), Guimarães et al. (2003), Lopes (1997a, 2002a, b), Lopes et al. (2002), Marcondes et al. (2003), Santos-Neto \& Lozovei (2008), Silva \& Lozovei (1999), Tissot \& Silva (2008)

x Barbosa et al. (1993), Consolim et al. (1993), Lopes et al. (1993), Lopes \& Lozovei (1996), Lopes (1997b, c, 2002a), Santos-Neto \& Lozovei (2008), Tissot \& Silva (2008)

x Consolim et al. (1993), Lopes \& Lozovei (1996), Lopes (2002a), Silva (2002), Tissot \& Silva (2008)

x Bona \& Navarro-Silva (2008), Guimarães et al. (2003), Tissot \& Silva (2008)

x Consolim et al. (1993)

x Lopes (2002a)

x Consolim et al. (1993), Lopes \& Lozovei (1996), Lopes (2002a), Marcondes et al. (2006)

x Barbosa et al. (1993, 2003), Bona \& Navarro-Silva (2008), Consolim et al. (1993), Guimarães et al. (2003), Marcondes et al. (2006), Paterno \& Marcondes (2004), Tissot \& Navarro-Silva (2004), Tissot \& Silva (2008)

x Consolim et al. (1993)

x Consolim et al. (1993), Marcondes et al. (2006)

x Marcondes et al. (2003), Tissot \& Silva (2008)

x Santos-Neto \& Lozovei (2008)

x Barbosa et al. (1993), Guimarães et al. (2003)

$\mathrm{x}$

$\mathrm{x}$

Consolim et al. (1993), Marcondes et al. (2006), Tissot \& Silva (2008)

x Lopes (2002a)

x Lopes (2002a), Teodoro et al. (1995)

x Tissot \& Silva (2008)

x Teodoro et al. (1995)

x Bona \& Navarro-Silva (2008), Tissot \& Navarro-Silva (2004), Tissot \& Silva (2008)

x consolim et al. (1993), Lopes (2002a), Teodoro et al. (1995)

x Tissot \& Silva (2008)

x Barbosa et al. (2003), Consolim et al. (1993), Lopes (2002a), Teodoro et al. (1995)

x Barbosa et al. (2003), Tissot \& Navarro-Silva (2004), Tissot \& Silva (2008)

x Consolim et al. (1993), Lopes (2002a), Teodoro et al. (1995)

x barbosa et al. (2003), Consolim et al. (1993), Guimarães et al. (2003), Lopes et al. (1995b, c), Lopes \& Lozovei (1996), Lopes (2002a), Teodoro et al. (1995), Tissot \& Navarro-Silva (2004)

$\mathrm{X}$
Barbosa et al. (2003), Lopes (2002a), Marcondes et al. (2006), Teodoro et al. (1995), Tissot \& Navarro-Silva (2004) 
Table 3. Continued..

\begin{tabular}{|c|c|c|c|}
\hline Species & Santa Catarina & Paraná & References \\
\hline Limatus durhamii Theobald 1901 & $\mathrm{x}$ & $\mathrm{x}$ & $\begin{array}{l}\text { Consolim et al. (1993), Guimarães et al. (2003), Lopes et al. } \\
\text { (1987, 1993, 1995a), Lopes (1997a, b, c, 1999, 2002a), } \\
\text { Marcondes et al. (2006), Paterno \& Marcondes (2004), } \\
\text { Silva (2002), Tissot \& Navarro-Silva (2004), Tissot \& Silva } \\
\text { (2008) }\end{array}$ \\
\hline Li. flavisetosus Oliveira Castro 1935 & & $\mathrm{x}$ & Tissot \& Silva (2008) \\
\hline Sabethes (Peytonulus) aurescens (Lutz 1905) & $\mathrm{x}$ & $\mathrm{x}$ & $\begin{array}{l}\text { Lozovei (1998, 2001), Marcondes \& Mafra (2003), Marcondes } \\
\text { et al. (2006), Paterno \& Marcondes (2004), Tissot \& Silva } \\
\text { (2008), Zequi \& Lopes (2001) }\end{array}$ \\
\hline Sa. (Pey.) undosus (Coquillett 1906) & & $\mathrm{x}$ & Tissot \& Silva (2008) \\
\hline Sa. (Sabethes) albiprivus Lutz 1903 & $\mathrm{x}$ & $\mathrm{x}$ & Guimarães et al. (2003), Paterno \& Marcondes (2004) \\
\hline Sa. (Sab.) batesi Lane \& Cerqueira 1942 & & $\mathrm{x}$ & Lozovei $(1998,2001)$ \\
\hline Sa. (Sab.) belisarioi Neiva 1908 & $\mathrm{x}$ & $\mathrm{x}$ & Consolim et al. (1993), Marcondes et al. (2006) \\
\hline Sa. (Sab.) purpureus (Theobald 1907) & & $\mathrm{x}$ & $\begin{array}{l}\text { Barbosa et al. (2003), Guimarães et al. (2003), Tissot \& Silva } \\
\text { (2008) }\end{array}$ \\
\hline $\begin{array}{l}\text { Sa. (Sabethoides) chloropterus } \\
\text { (Humbold 1820) }\end{array}$ & & $\mathrm{x}$ & Guimarães et al. (2003) \\
\hline Sa. (Sabethinus) identicus Dyar \& Knab 1907 & $\mathrm{x}$ & $\mathrm{x}$ & $\begin{array}{l}\text { Guimarães et al. (2003), Lopes (2002a), Marcondes et al. } \\
\text { (2006), Zequi \& Lopes (2001) }\end{array}$ \\
\hline Sa. (Sbn) indiogenes (Harbach 1994) & & $\mathrm{x}$ & Lopes $(2002 a)$ \\
\hline Sa. (Sbn) intermedius (Lutz 1904) & & $\mathrm{x}$ & Tissot \& Silva (2008) \\
\hline Sa. (Sbn) melanonymphe (Dyar 1924) & $\mathrm{x}$ & $\mathrm{x}$ & $\begin{array}{l}\text { Lozovei (1998, 2001), Lopes (2002a), Marcondes et al. (2003, } \\
\text { 2006), Zequi \& Lopes (2001) }\end{array}$ \\
\hline Sa. (Sbn) xhyphydes Harbach 1994 & & $\mathrm{x}$ & Tissot \& Silva (2008) \\
\hline $\begin{array}{l}\text { Wyeomyia (Dendromyia) personata } \\
\text { (Lutz 1904) }\end{array}$ & & $\mathrm{x}$ & Lozovei $(1998,2001)$ \\
\hline $\begin{array}{l}\text { Wy. (Exallomyia) tarsata } \\
\text { Lane \& Cerqueira } 1942\end{array}$ & $\mathrm{x}$ & & marcondes et al. (2003) \\
\hline $\begin{array}{l}\text { Wy. (Phoniomyia) antunesi } \\
\text { Lane \& Guimarães } 1937\end{array}$ & & $\mathrm{x}$ & Tissot \& Silva (2008) \\
\hline Wy. (Pho.) bourrouli Lutz 1905 & $\mathrm{x}$ & & marcondes et al. (2003), Paterno \& Marcondes (2004) \\
\hline Wy. (Pho.) davisi (Lane \& Cerqueira 1942) & $\mathrm{x}$ & $\mathrm{x}$ & $\begin{array}{l}\text { Bona \& Navarro-Silva (2008), Muller \& Marcondes (2006), } \\
\text { Paterno \& Marcondes (2004) }\end{array}$ \\
\hline Wy. (Pho.) edwardsi Lane \& Cerqueira 1942 & $\mathrm{x}$ & $\mathrm{x}$ & $\begin{array}{l}\text { Marcondes et al. (2003), Muller \& Marcondes (2006), Tissot } \\
\& \text { Silva (2008) }\end{array}$ \\
\hline Wy. (Pho.) fuscipes Edwards 1922 & $\mathrm{x}$ & & paterno \& Marcondes (2004) \\
\hline Wy. (Pho.) galvaoi (Correa \& Ramalho 1956) & $\mathrm{x}$ & $\mathrm{x}$ & $\begin{array}{l}\text { Bona \& Navarro-Silva (2008), Paterno \& Marcondes (2004), } \\
\text { Tissot \& Silva (2008) }\end{array}$ \\
\hline Wy. (Pho.) incaudata Root 1928 & $\mathrm{x}$ & $\mathrm{x}$ & $\begin{array}{l}\text { Bona \& Navarro-Silva (2008), Marcondes \& Paterno (2005), } \\
\text { Muller \& Marcondes (2006), Paterno \& Marcondes (2004) }\end{array}$ \\
\hline Wy. (Pho.) lopesi (Correa \& Ramalho 1956) & & $\mathrm{x}$ & Bona \& Navarro-Silva (2008) \\
\hline Wy. (Pho.) mystes Dyar 1924 & $\mathrm{x}$ & $\mathrm{x}$ & $\begin{array}{l}\text { Guimarães et al. (2003), Paterno \& Marcondes (2004), Tissot } \\
\& \text { Silva (2008) }\end{array}$ \\
\hline Wy. (Pho.) pallidoventer (Theobald 1907) & $\mathrm{x}$ & $\mathrm{x}$ & $\begin{array}{l}\text { Muller \& Marcondes (2006); Paterno \& Marcondes (2004); } \\
\text { Tissot \& Silva (2008) }\end{array}$ \\
\hline Wy. (Pho.) palmata (Lane \& Cerqueira 1942) & $\mathrm{x}$ & & paterno \& Marcondes (2004) \\
\hline Wy. (Pho.) pilicauda (Root 1928) & $\mathrm{x}$ & & Muller \& Marcondes (2006), Paterno \& Marcondes (2004) \\
\hline Wy. (Pho.) quasilongirostris (Theobald 1907) & $\mathrm{x}$ & $\mathrm{x}$ & $\begin{array}{l}\text { Barbosa et al. (2003), Guimarães et al. (2003), Marcondes } \\
\text { et al. (2003), Tissot \& Navarro-Silva (2004), Tissot \& Silva } \\
\text { (2008) }\end{array}$ \\
\hline $\begin{array}{l}\text { Wy. (Pho.) splendida } \\
\text { Bonne-Wepster \& Bonne } 1919\end{array}$ & $\mathrm{x}$ & & paterno \& Marcondes (2004) \\
\hline Wy. (Pho.) theobaldi (Lane \& Cerqueira 1942) & $\mathrm{x}$ & $\mathrm{x}$ & Bona \& Navarro-Silva (2008), Paterno \& Marcondes (2004) \\
\hline $\begin{array}{l}\text { Wy. (Pho.) tripartita } \\
\text { (Bonne-Webster \& Bonne 1921) }\end{array}$ & $\mathrm{x}$ & & marcondes et al. (2003), Muller \& Marcondes (2007) \\
\hline Wy. (Prosopolepis) confusa (Lutz, 1905) & & $\mathrm{x}$ & Guimarães et al. (2003) \\
\hline Wy. (Wyeomyia) abebela Dyar \& Knab 1908 & & $\mathrm{x}$ & Bona \& Navarro-Silva (2008) \\
\hline
\end{tabular}


Table 3. Continued...

\begin{tabular}{|c|c|c|c|}
\hline Species & Santa Catarina & Paraná & References \\
\hline Wy. (Wyo) limai Lane \& Cerqueira 1942 & $\mathrm{x}$ & $\mathrm{x}$ & $\begin{array}{l}\text { Lozovei (1998, 2001), Lopes (2002a), Marcondes \& Mafra } \\
\text { (2003, 2006), Tissot \& Silva (2008), Zequi \& Lopes (2001) }\end{array}$ \\
\hline Wy. (Wyo) lutzi (Costa Lima 1930) & $\mathrm{x}$ & $\mathrm{x}$ & Lozovei (1998, 2001), Marcondes et al. (2003) \\
\hline Wy. aporonoma Dyar \& Knab 1906 & & $\mathrm{x}$ & Guimarães et al. (2003) \\
\hline $\begin{array}{l}\text { Wy. argenteorostris (Bonne-Wepster \& Bonne } \\
\text { 1920) }\end{array}$ & $\mathrm{x}$ & & marcondes et al. (2003) \\
\hline Wy. cesari Del Ponte \& Cerqueira 1938 & $\mathrm{x}$ & & marcondes et al. (2003) \\
\hline $\begin{array}{l}\text { Wy. rooti (Del Ponte } 1939) \\
\text { Uranotaeniini }\end{array}$ & & $\mathrm{x}$ & Lopes (2002a), Zequi \& Lopes (2001) \\
\hline $\begin{array}{l}\text { Uranotaenia (Uranotaenia) apicalis Theobald } \\
1903\end{array}$ & & $\mathrm{x}$ & Lopes (2002) \\
\hline Ur. (Ura.) calosomata Dyar \& Knab 1907 & & $\mathrm{x}$ & Santos-Neto \& Lozovei (2008) \\
\hline Ur. (Ura.) geométrica Lutz 1901 & & $\mathrm{x}$ & Lopes (2002a), Lopes et al. (2002) \\
\hline Ur. (Ura.) lowii Theobald 1901 & & $\mathrm{x}$ & Silva (2002), Tissot \& Silva (2008) \\
\hline Ur. (Ura.) nataliae Lynch-Arribalzaga 1891 & & $\mathrm{x}$ & Santos-Neto \& Lozovei (2008), Silva (2002); \\
\hline $\begin{array}{l}\text { Ur. (Ura.) pulcherrima Lynch-Arribalzaga } \\
1891 \\
\text { Toxorhynchitini }\end{array}$ & & $\mathrm{x}$ & Lopes \& Lozovei (1995), Lopes (2002a) \\
\hline $\begin{array}{l}\text { Toxorhynchites (Ankylorhynchus) catharinensis } \\
\text { (Costa Lima, Guitton, \& Ferreira 1962) }\end{array}$ & $\mathrm{x}$ & & Marchon-Silva et al. (1996) \\
\hline $\begin{array}{l}\text { To. (Linchiella) bambusicolus (Lutz \& Neiva } \\
\text { 1913) }\end{array}$ & & $\mathrm{x}$ & Lozovei $(1998,2001)$ \\
\hline To. (Lyn.) pusillus (Costa Lima 1931) & & $\mathrm{x}$ & Lozovei (2001) \\
\hline To. (Lin.) theobaldi (Dyar \& Knab 1906) & & $\mathrm{x}$ & Silva \& Lozovei $(1996,1999)$ \\
\hline
\end{tabular}

Marcondes (2006) eight species new for Santa Catarina, besides three others for Paraná, which has been better studied, showing that the fauna of these southern Brazilian states is diversified and poorly known. This is specially marked for Wyeomyia (Phoniomyia), since 14 of the 21 known species were already reported from Santa Catarina. This group, which had only one new species (Wy. deanei Lourenço de Oliveira, 1983) described since 1956, certainly deserves a more thoroughly study. For instance, Anhembi arbovirus was isolated from Wy. pilicauda (Root 1928) in the littoral of São Paulo state (Souza Lopes et al. 1975).

\section{Acknowledgements}

CNPq granted a Ph. D. scholarship for GAM. Dr. Regiane Maria Tirone Menezes, of SUCEN/SES/SP, for checking some of the identifications and donation of related material (one female of Oc. hastatus/ oligopistus). Mr. Aristides Fernandes (Faculdade de Saúde Pública, Universidade de São Paulo), for checking some identifications. This study is part of the Project "Internal dynamics of rain forest: specificity of animal-plant interaction" within the Brazilian-German program "Mata Atlântica", and we acknowledge the financial support by BMBF (01LB0205) and CNPq (690143/01-0).

\section{References}

BARBOSA, O.C., TEODORO, U., LOZOVEI, A.L., SALVIA FILHO, V., SPINOSA, R.P., LIMA, E.M. \& FERREIRA, M.E.M.C. 1993. Nota sobre culicídeos adultos coletados na região sul do Brasil. Rev. Saúde Públ., 27(3):214-216.

BARBOSA, A.A., SILVA, M.A.N. \& CALADO, D. 2003. Atividade de Culicidae em remanescente florestal na região urbana de Curitiba (ParanáBrasil). Rev. Bras. Zool., 20(1):59-63.
BARBOSA, A.A., NAVARRO-SILVA, M.A. \& SALLUM, M.A. 2007. Mansonia (Mansonia) iguassuensis sp. nov. (Diptera: Culicidae) from Brasil. Zootaxa, 1527:1-8.

BONA, A.C.D. \& NAVARRO-SILVA, M.A. 2008. Diversidade de Culicidae durante os períodos crepusculares em bioma de Floresta Atlântica e paridade de Anopheles cruzii (Diptera: Culicidae). Rev. Bras. Zool., 25(1):40-48.

CARDOSO, J.C., CORSEUIL, E. \& BARATA, J.M.S. 2005. Culicinae (Diptera, Culicidae) ocorrentes no Estado do Rio Grande do Sul, Brasil. Rev. Bras. Entomol., 49(2):275-287.

COLLINS, L.E. \& BLACKWELL, A. 2000. The biology of Toxorhynchites mosquitoes and their potential as biocontrol agents. Biocontrol, 21(4):105-116.

CONSOLIM, J., PELLEGRINI, N.J.M. \& LUZ, E. 1993. Culicídeos (Diptera, Culicidae) do lago de Itaipú, Paraná, Brasil. Acta Biol. Par., 22:83-90.

CONSOLI, R.A.G.B. \& OLIVEIRA, R.L. 1994. Principais mosquitos de importância sanitária no Brasil. Fiocruz, Rio de Janeiro.

CORREA, R.R. \& RAMALHO, G.R. 1956. Revisão de Phoniomyia Theobald, 1903 (Diptera, Culicidae, Sabethini). Fol. Clin. Biol., 25:1-176.

FERREIRA, E. 1965. Distribuição geográfica dos anofelinos no Brasil e sua relação com o estado atual da erradicação da malária. Revta. Bras. Malar. Doen. Trop., 17:329-348.

FORATTINI, O.P. 2002. Culicidologia Médica. EDSP, São Paulo.

GUEDES, A.S., SOUZA, M.A., MACIEL, C.S. \& XAVIER, S.H. 1965. Catálogo ilustrado de mosquitos na Coleção do Instituto Nacional de Endemias Rurais. I. O gênero Psorophora Robineau-Desvoidy, 1827. Revta. Bras. Malariol. Doencas Trop., 17:3-24.

GUIMARÃES, A.E., MELlO, R.P., LOPES, C.M., ALENCAR, J. \& GENTILE, C. 1997. Prevalência de Anofelinos (Diptera: Culicidae) no Crepúsculo Vespertino em Áreas da Usina Hidrelétrica de Itaipu, no 
Município de Guaíra, Estado do Paraná, Brasil. Mem. Inst. Oswaldo Cruz, 92:745-754.

GUIMARÃES, A.E., LOPES, C.M., MELLO, R.P. \& ALENCAR, J. 2003. Ecologia de mosquitos (Diptera, Culicidae) em áreas do Parque Nacional do Iguaçu, Brasil. 1 - Distribuição por hábitat. Cad. Saúde Públ., 19(4):1107-1116.

HARBACH, R.E. 1994. The subgenus Sabethinus of Sabethes. (Diptera: Culicidae). Syst. Entomol., 19(3):207-234.

HEINEMANN, S.J., BELKIN, J.N. 1977. Collection records of the project "Mosquitoes of Middle America“. 9. Mexico (MEX, MF, MT, MX). Mosquito Syst. 9:483-535.

HUTCHINGS, R.S.G., SALLUM, M.A.M., FERREIRA, R.L.M. \& HUTCHINGS R.W. 2005. Mosquitoes of the Jaú National Park and their potential importance in Brazilian Amazonia. Med. Vet. Entomol.,19(4):428-441

LANE, J. 1953a. Neotropical Culicidae. Vol. 1. EDUSP, São Paulo.

LANE, J. 1953b. Neotropical Culicidae. Vol. 2. EDUSP, São Paulo.

LOPES, J., BORSATO, A.M. \& PIRES, M.A. 1987. Entomofauna da Mata Godoy. I. Culicidae (Dipetra) procriando-se em criadouros artificiais indroduzido na mata. Semina Ci. Biol./Saúde, 8(2):67-69.

LOPES, J., SILVA, M.A.N., BORSATO, A.M., OLIVEIRA, V.D.R.B. \& OLIVEIRA, F.J.A. 1993. Aedes (Stegomyia) aegypti L. e a culicideofauna associada em área urbana da região sul, Brasil. Rev. Saúde Públ., 27(5):326-333.

LOPES, J. \& LOZOVEI, A.L. 1995. Ecologia de mosquitos (Diptera: Culicidae) em criadouros naturais e artificiais de área rural do Norte do Estado do Paraná, Brasil. I - Coletas ao longo do leito de ribeirão. Rev. Saúde Públ., 29(3):183-191.

LOPES, J., SILVA, M.A.N., OLIVEIRA, V.D.R.B., BORSATO, A.M. \& BRAGA, M.C.P. 1995a. Ecologia de mosquitos (Diptera:Culicidae) em criadouros naturais e artificiais de área rural do Norte do Estado do Paraná, Brasil. III. Viabilização de recipientes como criadouro. Semina Ci. Biol./Saúde, 16(2):244-253.

LOPES, J., OLIVEIRA, F.J.A., OLIVEIRA, V.D.R.B. \& TONON, M.A.P. 1995b. Alterações na densidade populacional e diversidade de Culicidae (Diptera) na área urbana do município de Londrina, Estado do Paraná, Sul do Brasil em consequiência de modificações ambientais. Semina Ci. Biol./Saúde, 16(2):238-243.

LOPES, J., OLIVEIRA, V.D.R.B. \& OLIVEIRA, F.J.A. 1995c. Predominância de Mansonia titillans (Walker, 1848) (Diptera: Culicidae) na área urbana do município de Londrina-PR, Sul do Brasil. Semnia Ci. Biol./Saúde, 16(2):254-259.

LOPES, J. \& LOZOVEI, A.L. 1996. Ecologia de mosquitos (Diptera, Culicidae) em criadouros naturais e artificiais de área rural do Norte do Paraná, Brasil. II. Coletas com isca humana. Rev. Bras. Zool., 13(3):585-596.

LOPES, J. 1997a. Ecologia de mosquitos (Diptera: Culicidae) em criadouros naturais e artificiais de área rural do Norte do Estado do Paraná, Brasil. V. Coleta de larvas em recipientes artificiais instalados em mata ciliar. Rev. Saúde Públ., 31(4):370-377.

LOPES, J. 1997b. Ecologia de mosquitos (Diptera: Culicidae) em criadouros naturais e artificiais de área rural do Norte do Estado do Paraná, Brasil. VI Coletas de Larvas no Peridomicílio. Rev. Bras. Zool., 14(3):571-578.

LOPES, J. 1997c. Ecologia de mosquitos (Diptera: Culicidae) em criadouros naturais e artificiais de área rural do Norte do Estado do Paraná, Brasil. VII. Coexistência das espécies. Iheringia, Ser. Zool., 83:91-97.

LOPES, J. 1999. Ecologia de mosquitos (Diptera, Culicidae) em criadouros naturais e artificiais de área rural do norte do Paraná, Brasil. VIII. Influência das larvas predadoras (Toxorhynchites sp., Limatus durhamii e Culex bigoti) sobre a população de larvas de Culex quinquefasciatus e Culex eduardoi. Rev. Bras. Zool., 16(3):821-826.

LOPES, J. 2002a. Mosquitos (Diptera: Culicidae) da Região do Baixo Tibagi e suas adaptações a ambientes antropogênicos: causas e conseqüências. In A Bacia do Rio Tibagi. (M.E. Medri, E. Bianchini, O.A. Shibatta \& J.A. Pimenta, eds). Londrina, p. 327-351.
LOPES, J. 2002b. The occurrence of Aedes (Stegomyia) aegypti (Linnaeus) and Aedes (Stegomyia) albopictus (Skuse) in a rural área in Northern Paraná, Brazil and the associated Culicidae fauna. Rev. Bras. Zool., 19(4):1075-1079.

LOPES, J., ZEQUI, J.A.C., NUNES, V., OLIVEIRA, O., NETO, O.B.P.O. \& RODRIGUES, W. 2002. Immature Culicidae (Diptera) Collected from the Igapó Lake Located in the Urban area of Londrina, Paraná, Brazil. Braz. Arch. Biol. Technol., 45(4):565-471.

LOZOVEI, A.L. 1998. Mosquitos dendrícolas (Diptera, Culicidae) em internódios de taquara da floresta atlântica, serra do mar e do primeiro planalto, Paraná, Brasil. Braz. Arch. Biol. Technol., 41(4):501-508.

LOZOVEI, A.L. 2001. Microhabitats de mosquitos (Diptera, Culicidae) em internódios de taquara na Mata Atlântica, Paraná, Brasil. Iheringia, Sér. Zool., 90:3-13.

MARCHON-SILVA, V., LOURENÇO-DE-OLIVEIRA, R., ALMEIDA, M.D., SILVA-VASCONCELOS, A. \& COSTA, J. 1996. The Type Specimens of Mosquitoes (Diptera, Culicidae) Deposited in the Entomological Collection of the Instituto Oswaldo Cruz, Rio de Janeiro, Brazil. Mem. Inst. Oswaldo Cruz, 91(4):471-478.

MARCONDES, C.B. \& MAFRA, H. 2003. Nova técnica para o estudo da fauna de mosquitos (Diptera: Culicidae) em internódios de bambus, com resultados preliminares. Rev. Bras. Soc. Bra. Med. Trop., 36(6):763-764.

MARCONDES, C.B., FERNANDES, A., PATERNO, U., MÜLLER, G.A., PINHO, L.C. \& STRUFFALDI, D.V. 2003. New records of mosquitoes from the southern Brazilian states of Santa Catarina and Rio Grande do Sul, with 18 species new for the States (Diptera: Culicidae). Zootaxa, 347:1-6.

MARCONDES, C.B. \& PATERNO, U. 2005. Preliminary evidence of association between species of mosquitoes in Atlantic forest of Santa Catarina State, (Diptera: Culicidae). Rev. Bras. Soc. Bra. Med. Trop., 38(1):75-76.

MARCONDES, C.B., FERNANDES, A. \& MÜLLER, G.A. 2006. Mosquitoes (Diptera: Culicidae) near a reservoir in the Western part of the Brazilian State of Santa Catarina. Biota Neotrop., 6(3): http://www.biotaneotropica. org.br/v6n3/pt/abstract?inventory+bn02606032006 (ultimo acesso em 05/05/2008).

MARCONDES, C.B. 2007. Taxonomic changes: disprove or accept them. Trends Parasitol., 23:302-303.

MÉNDEZ, W., LIRIA, J., NAVARRO, J.C., GARCIÁ, C.Z., FREIER, J.E., SALAS, R., WEAVER, S.C. \& BARRERA, R. 2001. Spatial Dispersion of Adult Mosquitoes (Diptera: Culicidae) in a Sylvatic Focus of Venezuelan Equine Encephalitis Virus. J. Med. Entomol., 38(6):813-819.

MÜLLER, G.A. \& MARCONDES, C.B. 2006. Bromeliad-associated mosquitoes from Atlantic forest in Santa Catarina Island, southern Brazil (Diptera, Culicidae), with new records for the State of Santa Catarina. Iheringia, Sér. Zool., 96:315-319.

PATERNO, U. \& MARCONDES, C.B. 2004. Mosquitos antropofílicos de atividade matutina em Mata Atlântica em Florianópolis, Santa Catarina, Brasil (Diptera, Culicidae). Rev. Saúde Públ., 38(1):133-135.

PEREIRA, E.S., FERREIRA, R.L.M., HAMADA, N. \& LICHTWARDT, R.W. 2005. Trichomycete Fungi (Zygomycota) Associated with Mosquito Larvae (Diptera: Culicidae) in Natural and Artificial Habitats in Manaus, AM Brazil. Notrop. Entomol., 34(2):325-329.

PRADO, A. 1935. Contribuições ao conhecimento dos culicídeos de São Paulo. VI. Notas sobre os mosquitos originários das taquaras: Sabethoides intermedius (Lutz) e Megarhynus bambusicola Lutz \& Neiva. Mem. Instit. Butantan, 9:195-199.

RACHOU, R.G. \& FERRAZ, D.M. 1951. Contribuição ao conhecimento da distribuição geográfica dos anofelinos no Brasil: Estado de Santa Catarina. Rev. Bras. Malariol. Doen. Trop., 3(3):540-554.

REINERT, J.F. 1975. Mosquito generic and subgeneric abbreviations (Diptera: Culicidae). Mosquito System., 7:105-110.

REINERT, J.F. 2000. New classification for the composite genus Aedes (Diptera: Culicidae: Aedini), elevation of subgenus Ochlerotatus to generic rank, reclassification of the other subgenera, and notes 
Müller, G.A. et al.

on certain subgenera and species. J. Am. Mosquito Control Assoc., 16(3):175-188.

REINERT, J.F., HARBACH, R. \& KITCHING, I.J. 2004. Phylogeny and classification of Aedini (Diptera: Culicidae), based on morphological characters of all life stages. Zool. J. Linn. Soc., 142:289-368.

REINERT, J.F., HARBACH, R. \& SALLUM, M.A.M. 2005. Checklist of aedine mosquito species (Diptera, Culicidae, Aedini) occurring in Middle and South America (south of the United States) reflecting current generic and subgeneric status. Rev. Bras. Entomol., 49(2):249-252.

SANT'ANA, A.L. \& LOZOVEI, A.L. 2001. Influência do ciclo lunar na captura de Aedes scapularis (Diptera, Culicidae) na mata Atlântica do Parana. Iheringia, Ser. Zool., 90:175-182.

SANTOS-NETO, L.G. \& LOZOVEI, A.L. 2008. Aspectos ecológicos de Anopheles cruzii e Culex ribeirensis (Diptera, Culicidae) da Mata Atlântica de Morretes, Paraná, Brasil. Rev. Bras. Entomol., 52(1):105-111.

SILVA, A.M. \& LOZOVEI, A.L. 1996. Criadouros de imaturos de mosquitos (Diptera, Culicidae) introduzidos em mata preservada na área urbana de Curitiba, Paraná, Brasil. Revta. Bras. Zool., 13(4):1023-1042.

SILVA, M.A.N. \& LOZOVEI, A.L.1999. Ocorrência de Haemagogus (Conopostegus) leucocelaenus (Dyar \& Shannon) e Toxorhynchites (Lynchiella) theobaldi (Dyar \& Knab) em ocos de árvore em capão de mata, Curitiba, Paraná, Brasil. Rev. Bras. Zool., 16 (1):257-267.

SILVA, A.M. \& MENEZES, R.M.T. 1996. Encontro de Aedes scapularis (Diptera: Culicidae) em criadouro artificial em localidade da região Sul do Brasil. Rev. Saúde Públ., 30(1):103-104.

SILVA, M.A.N. \& LOZOVEI, A.L. 1999. Ocorrência de Haemagogus (Conopostegus) leucocelaenus (Dyar \& Shannon) e Toxorhynchites (Lynchiella) theobaldi (Dyar \& Knab) em oco de árvore em capão de mata, Curitiba, Paraná, Brasil. Rev. Bras. Zool., 16(1):257-267.
SILVA, A.M. 2002. Imaturos de mosquito (Diptera, Culicidae) de áreas urbana e rural no norte do Estado do Paraná, Brasil. Iheringia, Sér. Zool., 92(4):31-36.

SOUZA LOPES, O., SACCHETTA, L.A., FONSECA, I.E. \& LACERDA, J.P. 1975 Bertioga (Guama group) and Anhembi (Bunyamwera group), two new arboviruses isolated in São Paulo, Brazil. Am. J. Trop. Med. Hyg., 4(1):131-134.

TEODORO, U., GUILHERME, A.L.F., LOZOVEI, V., FILHO, V.L.S., FUKUSHIGUE, Y., SPINOSA, R.P., FERREIRA, M.E.M.C., BARBOSA, O.C. \& LIMA, E.M. 1995. Culicídeos do Lago de Itaipu, no Rio Paraná, Sul do Brasil. Rev. Saúde Públ., 29(1):6-14.

TISSOT, A.C. \& NAVARRO-SILVA, M.A. 2004. Preferência por hospedeiro e estratificação de Culicidae (Diptera) em área de remanescente florestal do Parque Regional do Iguaçu, Curitiba, Paraná, Brasil. Rev. Brás. Zool., 21(4):877-886.

TISSOT, A.C. \& SILVA, M.A.N. 2008. Lista das espécies de Culicidae (Diptera) depositadas na Coleção de Entomologia Pe. J. S. Moure. Rev. Bras. Entomol., 52(2):263-268.

VELOSO, H.P., Rangel Filho, A.L. \& Lima, J.C.A. 1991. Classificação da vegetação brasileira, adaptada a um sistema universal. IBGE, Rio de Janeiro.

ZEQUI, J.A.C. \& LOPES, J. 2001. Culicideofauna (Diptera) encontrada em entrenós de taquara de uma mata residual na área urbana de Londrina, Paraná, Brasil. Rev. Bras. Zool., 18:429-438.

Data Received 07/07/08 Revised 31/10/08 Accepted 04/11/08 